\title{
Practice guidelines for propofol sedation by non-anesthesiologists : the Korean Society of Anesthesiologists Task Force recommendations on propofol sedation
}

\author{
Hyun Kang ${ }^{1}$, Duk Kyung Kim², Yong-Seon Choi ${ }^{3}$, Young-Chul Yoo ${ }^{3}$, and \\ Hyun Sik Chung ${ }^{4}$
}

Department of Anesthesiology and Pain Medicine, ${ }^{1}$ Chung-Ang University College of Medicine, ${ }^{2}$ Samsung Medical Center, Sungkyunkwan University School of Medicine, ${ }^{3}$ Severance Hospital, Yonsei University College of Medicine, ${ }^{4}$ Seoul St. Mary's Hospital, College of Medicine, The Catholic University of Korea, Seoul, Korea

In South Korea, as in many other countries, propofol sedation is performed by practitioners across a broad range of specialties in our country. However, this has led to significant variation in propofol sedation practices, as shown in a series of reports by the Korean Society of Anesthesiologists (KSA). This has led the KSA to develop a set of evidence-based practical guidelines for propofol sedation by non-anesthesiologists. Here, we provide a set of recommendations for propofol sedation, with the aim of ensuring patient safety in a variety of clinical settings. The subjects of the guidelines are patients aged $\geq 18$ years who were receiving diagnostic or therapeutic procedures under propofol sedation in a variety of hospital classes. The committee developed the guidelines via a de novo method, using key questions created across 10 sub-themes for data collection as well as evidence from the literature. In addition, meta-analyses were performed for three key questions. Recommendations were made based on the available evidence, and graded according to the modified Grading of Recommendations Assessment, Development and Evaluation system. Draft guidelines were scrutinized and discussed by advisory panels, and agreement was achieved via the Delphi consensus process. The guidelines contain 33 recommendations that have been endorsed by the KSA Executive Committee. These guidelines are not a legal standard of care and are not absolute requirements; rather they are recommendations that may be adopted, modified, or rejected according to clinical considerations.

Key Words: Conscious sedation, Deep sedation, Guideline, Propofol.

Corresponding author: Duk Kyung Kim, M.D., Ph.D.

Department of Anesthesiology and Pain Medicine, Samsung Medical Center, Sungkyunkwan University School of Medicine, 81, Irwon-ro, Gangnam-gu, Seoul 06351, Korea

Tel: 82-2-3410-0343, Fax: 82-2-3410-6626, Email: dikei@hanmail.net

ORCID: http://orcid.org/0000-0002-6555-2100

This practice guidelines summarizes the complete contents of "Practice Guideline for Propofol Sedation by Non-anesthesiologists" which published by Korea Health Industry Development Institute (KHIDI) in July 2016. For more detailed information and references, readers are encouraged to read the complete contents at the web page of "http://www.anesthesia.or.kr/guidelines/". This publication is only available in Korean.

Received: June 17, 2016. Accepted: June 21, 2016.

Korean J Anesthesiol 2016 December 69(6): 545-554

https://doi.org/10.4097/kjae.2016.69.6.545

(c) This is an open-access article distributed under the terms of the Creative Commons Attribution Non-Commercial License (http://creativecommons.org/ licenses/by-nc/4.0/), which permits unrestricted non-commercial use, distribution, and reproduction in any medium, provided the original work is properly cited. 


\section{Introduction}

Because of the favorable pharmacokinetic profile of propofol (i.e., rapid onset of action and short recovery time), it has been widely used for sedation by practitioners across a broad range of specialties. In contrast to the agents used for traditional sedation (i.e., benzodiazepines and/or opioids), propofol can easily lead to deep sedation or even general anesthesia requiring cardiopulmonary support. A series of reports by the Korean Society of Anesthesiologists (KSA) found that the number of disputed medical cases involving propofol sedation has increased in recent years, and the magnitude of injuries associated with propofol sedation is similar to those with general anesthesia [1,2]. Significant variation in propofol sedation practices was also found across different types of procedures, clinical specialties, and hospital classes.

In response to these issues, the KSA developed evidence-based practical guidelines for propofol sedation by non-anesthesiologists (anesthesiologists have specific expertise in the pharmacology, physiology, and clinical management of patients receiving propofol sedation). Here, we describe a set of recommendations for ensuring patient safety in a variety of clinical settings. These guidelines were formulated via a de novo method, which involved a systematic review of the available evidence and a synthesis of expert opinion. These guidelines are neither legal standards of care nor absolute requirements; therefore, clinical considerations may lead a practitioner to take a course of action that varies from these guidelines.

\section{Methodology}

\section{Definitions of 'propofol sedation' and 'level of sedation'}

Propofol sedation is defined as a technique of administering propofol with or without other sedatives/analgesics to induce a state that allows patients to tolerate unpleasant procedures while maintaining cardiorespiratory function. Although the level of sedation can be assessed using different scales, here we followed the definitions of levels of sedation and anesthesia given by the American Society of Anesthesiologists (ASA) (Table 1) [3].

\section{Scope}

The subjects of this work were adult patients (i.e., aged $\geq 18$ years) receiving diagnostic or therapeutic procedures under propofol sedation in a variety of hospital classes (including primary, secondary, and tertiary healthcare classes). These guidelines were originally intended to provide useful information and guidance for all of the Korean non-anesthesiologist practitioners who perform propofol sedation. However, below we list some situations that we excluded from the scope of the guidelines:

- sedation in intensive care units;

- sedation for patients with artificial airways;

- sedation for dental procedures;

- palliative sedation at the end-of-life;

- and intended use of propofol for general anesthesia.

\section{Methods used to develop guidelines}

Fig. 1 shows a summary of the process used to develop these guidelines. The KSA appointed a Task Force of five members to determine the scope of the guidelines, create key questions in 10 sub-themes for data collection, systemically review the published data, make draft recommendations with supporting evidence, and draft the guidelines.

Because there were no existing evidence-based guidelines consistent to our scope, these guidelines were developed de novo. Two similar sets of guidelines for propofol sedation did exist; however, their scopes were limited to gastrointestinal (GI) endoscopy $[4,5]$.

The Task Force team systematically collected and reviewed the international and domestic published literature using the PubMed, Cochrane, Embase, Scopus, KoreaMed, and Google Scholar databases. The literature was limited to publications before December 31, 2015, studies performed on adult patients, and studies published in the English or Korean languages.

Table 1. Levels of Sedation and Anesthesia as Defined by the ASA [3]

\begin{tabular}{|c|c|c|c|c|}
\hline & Minimal sedation & Moderate sedation & Deep sedation & General anesthesia \\
\hline Responsiveness & $\begin{array}{c}\text { Normal response to } \\
\text { verbal stimulation }\end{array}$ & $\begin{array}{l}\text { Purposeful response to } \\
\text { verbal or tactile } \\
\text { stimulation }\end{array}$ & $\begin{array}{l}\text { Purposeful response after } \\
\text { repeated or painful } \\
\text { stimulation }\end{array}$ & $\begin{array}{l}\text { Unarousable even with } \\
\text { painful stimulus }\end{array}$ \\
\hline Airway & Unaffected & No intervention required & $\begin{array}{l}\text { Intervention may be } \\
\text { required }\end{array}$ & $\begin{array}{l}\text { Intervention often } \\
\text { required }\end{array}$ \\
\hline Spontaneous ventilation & Unaffected & Adequate & May be inadequate & Frequently inadequate \\
\hline Cardiovascular function & Unaffected & Usually maintained & Usually maintained & May be impaired \\
\hline
\end{tabular}

American Society of Anesthesiologists Task Force on Sedation and Analgesia by Non-Anesthesiologists. Practice guidelines for sedation and analgesia by non-anesthesiologists. Anesthesiology 2002; 96: 1004-17. ASA: American Society of Anesthesiologists. 
When a more recent systematic review or meta-analysis study was found, studies with a lower level of evidence were excluded. When there was no existing systematic review or meta-analysis of the high-ranked key questions (which were selected based on 9-point Likert scale), additional meta-analyses were performed (see recommendations 19, 21, 22).

\section{Level of evidence and grade of recommendation}

The evidence levels used in these guidelines were determined

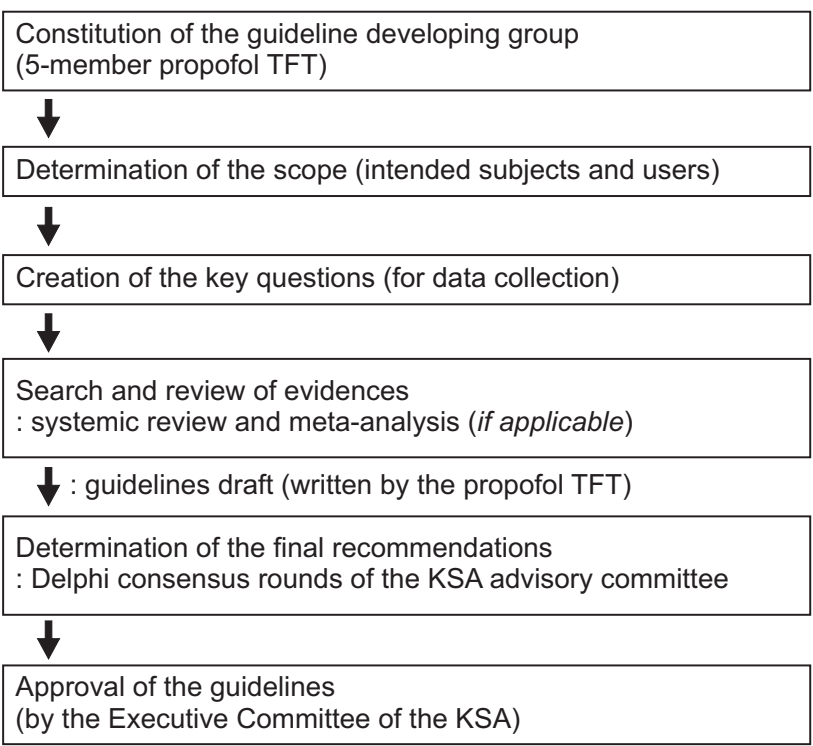

Fig. 1. Schematic diagram of the overall process used to determine the guideline. TFT: Task Force tram, KSA: Korean Society of Anesthesiologists. using the University of Michigan Health System (UMHS) method, whereby the validity of an individual study was only based on an assessment of the study design (Table 2). The strength of a recommendation was determined according to the modified Grading of Recommendations Assessment, Development and Evaluation (GRADE) system (Table 3 ). In addition to the level of evidence, the results of the studies were considered based on aspects of the clinical usefulness and trade-offs between health outcomes and patient benefit on the one hand, and the risks, difficulties, and costs on the other.

\section{Review and guidelines determination process}

The guidelines were drafted with recommendations that were initially suggested by the Task Force team, and were individually reviewed by all of the advisory panels prior to approval at a consensus meeting; the Delphi method was used to arrive at a consensus [6]. The advisory committee for the Delphi consensus process comprised 14 panelists who were members of the KSA Executive Committee. The agreement for each recommendation was graded according to the following response scale: 1-3, disagree; 4-6, uncertain, 7-9, agree. If over 50\% of the panel replied with a response of 7-9, a panel consensus was considered achieved. Of the 34 total recommendations, a consensus was reached on 32 of them in the first round, one recommendation required additional revision (recommendations 15) in a second round, and the remaining recommendation was eventually discarded. The selected recommendations were incorporated into the final draft of the guidelines, and approved by the KSA Executive Committee.

Table 2. Definition of the Levels of Evidence in the Guidelines

\begin{tabular}{cl}
\hline Level of evidence & \multicolumn{1}{c}{ Definition } \\
\hline A & Evidence obtained from at least one randomized controlled trial or systematic review/meta-analysis \\
B & Evidence obtained from at least one well-designed case-control or cohort trial without randomization \\
C & Evidence obtained from observational trials (case reports or case series) \\
D & Evidence obtained from opinion of expert panels \\
\hline
\end{tabular}

Table 3. Definition of the Recommendation Grade in the Guidelines

\begin{tabular}{cll}
\hline $\begin{array}{c}\text { Grade of } \\
\text { recommendation }\end{array}$ & \multicolumn{1}{c}{ Definition } & Description \\
\hline Class 1 & High level of evidence (A), substantial net benefit, and high clinical applicability & Is recommended \\
Class IIa & Confident level of evidence (B) and net benefit, and high or moderate clinical applicability & Should be considered \\
Class IIb & Uncertain level of evidence (C or D) and net benefit, and high or moderate clinical applicability & May be considered \\
Class III & Uncertain level of evidence (C or D), maybe harmful, and low clinical applicability & Is not recommended \\
\hline
\end{tabular}

Although the class of recommendations was mainly determined based on the level of evidence, some could be downgraded or upgraded by the advisory panels, based on further consideration of net benefit or clinical applicability. 


\section{Independence of support and editing}

During the development of these guidelines, there was no external influence from any other academies, institutions, or interest groups. During the planning of the guidelines, members of relevant academies, institutions, or organizations (although they may have been intended users of the guidelines) were not allowed to participate in the development of these guidelines because of potential significant and direct interests in the outcome of the guidelines. In 2015, the Korean Medical Association (KMA) attempted to develop a similar set of guidelines (i.e., practical guidelines for propofol sedation by non-anesthesiologist practitioners in a primary healthcare class) with participation from various stakeholders. However, those guidelines were influenced by these interest groups, and as such, many of the recommendations appeared arbitrary and were criticized for lacking impartiality. All of the members who participated in the development of these guidelines signed agreements confirming that there were no conflicts of interest in connection with this work.

\section{Funding source}

The development of these guidelines was supported by a grant from the Clinical Research Infrastructure Composition Project (project title: Patient Safety Policy Research) from the Korean Ministry of Health and Welfare.

\section{Release of the guidelines and plan for updates}

The (more detailed) Korean language version of the guidelines was released and published in June 2016 on the KSA website (http://www.anesthesia.or.kr/guidelines). Further documentation related to propofol sedation is also available on the website including informed consent forms, pre-sedation evaluation records, sedation records, and post-sedation recovery records. Given the rapid pace of changes in this field, updates to the guidelines are planned to be released at intervals of 3-5 years.

\section{Contents}

\section{Propofol sedation vs. traditional sedation}

Recommendation 1: Compared to traditional sedation, propofol sedation presents similar rates of adverse cardiorespiratory effects in low-or average-risk patients undergoing GI endoscopic procedures (Evidence level: A, Recommendation grade: Class I).

Recommendation 2: The above statement is valid for procedures with a relatively short duration or for less invasive proce- dures in other clinical fields (Evidence level: C, Recommendation grade: Class IIa).

Recommendation 3: In terms of cost-effectiveness, propofol sedation is superior to traditional sedation (Evidence level: D, Recommendation grade: Class III).

\section{Commentary}

Several meta-analyses [7-11] have shown clinical advantages of propofol sedation for GI endoscopy over traditional sedation with similar rates of adverse events (apnea, hypoxemia, hypotension, or arrhythmia). However, these results should be carefully interpreted in that all of the included studies were performed in a GI endoscopy setting, and most sedation cases were well controlled (e.g., independent monitoring of relatively well-trained medical personnel and adequate levels of intra-procedural monitoring). Therefore, such results may not be directly applicable to other clinical fields or poorly controlled clinical environments. In addition, considering the pharmacological characteristics of propofol, it is clear that poorly controlled propofol sedation carries elevated risks of adverse cardiorespiratory effects compared to traditional sedation $[12,13]$.

\section{Pre-procedural preparation and assessment}

Recommendation 4: In patients who chronically receive benzodiazepines, barbiturates, anticonvulsants, or alcohol, the dose requirements of propofol may be increased (Evidence level: $C$, Recommendation grade: Class IIb).

Recommendation 5: Propofol sedation in the first trimester should be avoided if at all possible (Evidence level: C, Recommendation grade: Class IIa).

Recommendation 6: No interruption of breastfeeding is required following propofol sedation (Evidence level: B, Recommendation grade: Class IIa).

\section{Commentary}

Propofol is classified as a 'category B' drug by the United States Food and Drug Administration, and safety in the first trimester has not been well studied. In addition, invasive procedures should be deferred until the second trimester whenever possible, and should always have a strong indication with a careful assessment of risk [14]. Although propofol may be excreted in breast milk, at clinical doses the quantity is too small to have significant effects on infants.

Recommendation 7: Propofol can be safely used in adult patients with allergies to eggs (Evidence level: C, Recommendation grade: Class IIb).

Recommendation 8: In propofol sedation for elective procedures, the same level of fasting time is required as for general anes- 
thesia (Evidence level: B, Recommendation grade: Class I).

Recommendation 9: The advantages and potential risks of propofol sedation as well as alternative options (traditional sedation or sedation by anesthesiologists) should be provided. Activities requiring a high level of psychomotor function (e.g., driving, operating heavy machinery, or engaging in legally binding decisions) should be refrained from for a period of time. Informed consent for propofol sedation should also be obtained from the patient or his/her legal representative (Evidence level: C, Recommendation grade: Class IIa).

Recommendation 10: Intravenous access is required for propofol sedation and should be maintained using a catheter until full patient recovery (Evidence level: $C$, Recommendation grade: Class IIa).

\section{Commentary}

Allergic reactions to propofol are extremely rare, with an estimated incidence of 1 in 60,000 exposures [15]. Furthermore, patients who are allergic to eggs typically react to proteins from the egg white but not to egg lecithin (from the yolk), which is used in propofol. Therefore, the practice of choosing alternatives to propofol in patients with known allergies to eggs is not evidence based approach [16].

As suggested by the sedation guidelines of the American College of Emergency Physicians [17], Recommendation 8 is mainly based on the extrapolation of general anesthesia cases in the operating rooms. However, because patient safety was the highest priority when formulating the guidelines, the KSA strongly supports the same fasting time as that used for general anesthesia (i.e., $2 \mathrm{~h}$ of fasting for clear liquids and $6 \mathrm{~h}$ of fasting for milk or solids) in propofol sedation for elective cases.

\section{Sedation personnel}

Recommendation 11: During propofol sedation, patients should be continuously monitored by a medical person who has patient sedation as his/her sole task (Evidence level: B, Recommendation grade: Class IIa).

Recommendation 12: Indications for propofol sedation by anesthesiologists:

(1) American Society of Anesthesiologists (ASA) physical status $\geq I I I$

(2) Cases where prolonged procedure $(\geq 2 h)$ is anticipated or deep sedation is required in highly invasive procedures;

(3) Patients with potentially difficult airways for intubation or mask ventilation;

(4) Patients with a known history of side effects in previous sedation or anesthesia (Evidence level: C, Recommendation grade: Class IIa); and

(5) Elderly patients aged $\geq 70$ years (Evidence level: D, Recom- mendation grade: Class IIb).

\section{Commentary}

These guidelines suggest that the team for propofol sedation should consist of a 'medical doctor in charge of sedation' and 'monitoring medical personnel'. In other words, it is recommended that patients should be continuously monitored by a person solely dedicated to propofol sedation.

However, in Korea, a considerable number of propofol sedations have been performed without independent monitoring by medical personnel [18]. Although the KSA fully supports the necessity of an additional staff member solely for propofol sedation for all procedures and patients, Recommendation 11 is graded as 'class IIb' due to low clinical applicability for prohibiting brief interruptions of monitoring by medical personnel. Allowance for brief interruptions of monitoring due to assistance for procedures (particularly in low- or average-risk patients undergoing simple procedures) is a matter of debate, particularly in GI endoscopy [5].

Another contentious issue is the safety of propofol sedation by non-anesthesiologists in patients of ASA physical status III. There is little debate regarding patients of ASA physical status IV. Increased ASA class is associated with increased rates of sedation-related adverse events; this has been established both via large retrospective studies [19] and prospective studies [20]. However, as a cut-off criterion for anesthesiology assistance, it remains vague. Some GI endoscopy guidelines have expressed doubts over the necessity of anesthesiology assistance in patients of ASA physical status III undergoing relatively simple procedures [21,22].

\section{Intra-procedural monitoring and equipment}

Recommendation 14: All of the patients undergoing propofol sedation should be monitored by continuous pulse oximetry and automated noninvasive blood pressure measurements during sedation, with intervals of a minimum of $5 \mathrm{~min}$. (Evidence level: $B$, Recommendation grade: Class I).

Recommendation 15: During propofol sedation, capnographic monitoring of respiratory activity is required in specific situations including high-risk patients, intended deep sedation, and long procedures ( $\geq 2$ h) (Evidence level: B, Recommendation grade: Class IIa).

Recommendation 16: Continuous electrocardiography is required in patients with a history of cardiovascular and/or pulmonary disease (Evidence level: C, Recommendation grade: Class IIa).

Recommendation 17: All of the cases of deep sedation and specific cases of moderate sedation (i.e., sedation for patients with cardiovascular and/or pulmonary disease) should only peformed 
in a setting adequately equipped with a defibrillator (Evidence level: C, Recommendation grade: Class IIa).

Recommendation 18: Continuous supplemental oxygen is indicated in every case of propofol sedation, unless specifically contraindicated for a particular patient or procedure (Evidence level: A, Recommendation grade: Class I).

\section{Commentary}

Recommendation 15 is the only one in which we did not reach a consensus in the first round of the Delphi consensus process. After upgrading 'class $I I a$ ' from 'class $I I b$ ', we reached a consensus in the second round. Societies of GI endoscopists have recommended that the use of capnography should not mandatory, even with propofol sedation $[4,5,21,22]$. The evidence for this stems from several randomized controlled trials $[23,24]$, in which use of capnography provided early detection of hypoxemia, but failed to show any difference in clinical outcomes. However, since 2010, the ASA officially stated that capnographic monitoring of respiratory activity should be mandatory during moderate or deep sedation [25]. The KSA also takes a favorable position about the utility of capnography during propofol sedation in that visual inspection of respiratory activity (i.e., another possible option) is less reliable and not always possible.

\section{Level of sedation}

Recommendation 19: If possible, the level of sedation should be maintained below deep sedation (Evidence level: A, Recommendation grade: Class IIa).

Recommendation 20: There are no pre-determined dose of propofol and uniform administration method depending on procedures. Prior to every sedation, the target level of sedation should be determined. During sedation, the dose of propofol should be continuously titrated to maintain the target level of sedation based on regular monitoring of the depth of sedation (Evidence level: D, Recommendation grade: Class IIb).

\section{Commentary}

Our meta-analysis indicates that, compared to deep sedation, moderate sedation is associated with a decreased risk of desaturation, with similar efficacy profiles (patient and doctor satisfactions, as well as recovery time), as well as risk of recall (Table 4). Therefore, unnecessary deep sedation should be avoided.

Because of a lack of analgesic properties of propofol, deep sedation is typically required for painful procedures when propofol is used alone for sedation. In such cases, moderate sedation is more likely to be achieved by a combination of propofol and

Table 4. Summary of Meta-analysis

\begin{tabular}{|c|c|c|c|c|c|c|}
\hline \multirow{3}{*}{ Parameters } & \multicolumn{4}{|c|}{ Summary estimates } & \multirow{2}{*}{\multicolumn{2}{|c|}{ Heterogeneity }} \\
\hline & \multicolumn{3}{|c|}{ Continuous variables } & \multirow{2}{*}{$\begin{array}{c}\text { Categorical variables } \\
\text { Weighted mean } \\
\text { difference }(95 \% \mathrm{CI})\end{array}$} & & \\
\hline & $\begin{array}{c}\text { No. of } \\
\text { estimates }\end{array}$ & $\begin{array}{l}\text { Risk ratio } \\
(95 \% \text { CI })\end{array}$ & $\begin{array}{c}\text { No. of } \\
\text { estimates }\end{array}$ & & $I^{2}(\%)$ & $\mathrm{P}_{\mathrm{chi}^{2}}$ \\
\hline \multicolumn{7}{|c|}{ Recommendation 21: Combination therapy vs. mono-therapy } \\
\hline Respiratory complication & 15 & $1.12(0.73,1.71)$ & & & 52 & 0.010 \\
\hline Hypotension & 12 & $1.23(0.71,2.14)$ & & & 65 & 0.001 \\
\hline Total propofol dose & & & 18 & $0.79(-1.03,-0.55)$ & 83 & $<0.001$ \\
\hline Arrhythmia & 10 & $1.55(0.97,2.48)$ & & & 15 & 0.301 \\
\hline Patients satisfaction & & & 8 & $0.13(-0.26,0.52)$ & 90 & $<0.001$ \\
\hline Doctor satisfaction & & & 5 & $0.01(-0.15,0.17)$ & 0 & 0.779 \\
\hline Recovery time & & & 7 & $0.27(-0.46,0.99)$ & 96 & $<0.001$ \\
\hline Procedure time & & & 14 & $0.03(-0.08,0.14)$ & 0 & 0.489 \\
\hline \multicolumn{7}{|c|}{ Recommendation 22: Intermittent bolus injection vs. continuous infusion } \\
\hline Total propofol dose & & & 6 & $-0.74(-1.05,-0.44)$ & 63 & 0.022 \\
\hline Number of intervention & & & 2 & $1.82(1.30,2.35)$ & 0 & 0.721 \\
\hline Sedation time & & & 3 & $-3.55(-5.61,1.48)$ & 9 & 0.331 \\
\hline Recovery time & & & 5 & $-2.33(-3.55,-1.11)$ & 45 & 0.141 \\
\hline Doctor satisfaction & & & 3 & $-0.39(-1.06,0.28)$ & 86 & $<0.001$ \\
\hline Cardiorespiratory complications & 6 & $0.86(0.48,1.56)$ & & & 0 & 0.972 \\
\hline \multicolumn{7}{|c|}{ Recommendation 19: Moderate sedation vs. deep sedation } \\
\hline Patient satisfaction & & & 3 & $0.94(0.86,1.04)$ & 65 & 0.059 \\
\hline Doctor satisfaction & & & 2 & $0.35(0.02,6.95)$ & 100 & $<0.001$ \\
\hline Recall & 2 & $5.82(0.51,66.48)$ & & & 60 & 0.108 \\
\hline Desaturation & 3 & $0.18(0.03,0.99)$ & & & 0 & 0.482 \\
\hline Recovery time & & & 3 & $-6.77(-16.21,2.67)$ & 99 & $<0.001$ \\
\hline
\end{tabular}


analgesics [26]. Recommendation 20 was developed based on considerations that different patients may require different levels of sedation for a given procedure, and may attain varying levels of sedation during that procedure.

\section{Propofol administration techniques}

Recommendation 21: In GI endoscopic procedures, propofol combination therapy with other agents is as effective and safe as propofol monotherapy (Evidence level: A, Recommendation grade: Class IIa).

\section{Commentary}

We performed a meta-analysis of randomized controlled trials comparing propofol combination therapy with propofol monotherapy for GI endoscopic procedures, both in terms of efficacy and safety (Table 4). Our results show that propofol combination therapy may enable a significant reduction in the total dose of propofol, but without the benefits of reduced risk of cardiopulmonary complications (apnea, hypoxemia, hypotension, and arrhythmia) compared to propofol monotherapy. Thus, both therapies can be safely used based on the practitioner's clinical judgment.

In severely agitated patients or in patients undergoing painful procedures, a combination of propofol with analgesics and/or other sedatives may reduce the required dose of propofol, thereby resulting in a lower level of sedation [26,27]. Although such techniques may also reduce the dose-related adverse effects of propofol, the doses of each drug should be carefully controlled due to potential synergistic effects on respiratory depression.

Recommendation 22: Intermittent bolus injection, targetcontrolled infusion (TCI), and manually controlled infusion (MCI) present similar safety profiles. Thus, they may all be applicable based on the practitioner's clinical judgment (Evidence level: A, Recommendation grade: Class I).

\section{Commentary}

We performed a meta-analysis of randomized controlled trials comparing intermittent bolus injection with continuous infusion (TCI and MCI) for propofol sedation, both in terms of efficacy (total propofol dose, number of intervention, sedation time, recovery time, doctor's satisfaction) and safety (cardiorespiratory complications) (Table 4). Our results show that intermittent bolus injection may significantly reduce the total dose of propofol, sedation time, and recovery time, but without the benefits of reduced risk of cardiopulmonary complications compared to continuous infusion. In addition, more frequent interventions were required by the practitioner during the use of intermittent bolus injection compared to continuous infusion.
Other meta-analyses included randomized controlled trials for sedation as well as general anesthesia, and showed that TCI is associated with higher total doses of propofol than MCI, but that both techniques entail similar risks of adverse events [28]. Thus, all of the three administration techniques can be safely applied based on the practitioner's clinical judgment.

\section{Surveillance during recovery and discharge}

Recommendation 23: A minimum recovery time of $30 \mathrm{~min}$ is required following propofol sedation (Evidence level: B, Recommendation grade: Class IIa).

Recommendation 24: In propofol sedation, the medical personnel in charge of recovery require the same level of qualification as the monitoring medical personnel (Evidence level: D, Recommendation grade: Class IIb).

Recommendation 25: Discharge criteria, such as the Modified Aldrete score or postanesthetic discharge scoring system (PADSS), should be applied when discharging patients following propofol sedation (Evidence level: A, Recommendation grade: Class I).

Recommendation 26: Because existing discharge criteria do not adequately evaluate psychomotor performance, discharged patients should be in the company of a responsible adult. If possible, propofol sedation should be avoided for outpatients without a companion (Evidence level: C, Recommendation grade: Class IIb).

Recommendation 27: Prior to discharge from hospital, patients should be instructed to refrain from driving for $24 \mathrm{~h}$ (Evidence level: C, Recommendation grade: Class IIb).

\section{Commentary}

The rationale for the minimum recovery time of $30 \mathrm{~min}$ is that most serious sedation-related complications occur within $30 \mathrm{~min}$ from completion of the procedure [29]. Compared to the Modified Aldrete score, PADSS is more suitable for propofol sedation, particularly in an outpatient setting [5]. Recent data suggest that propofol sedation results in improved psychomotor recovery compared to traditional sedation, with 1-6 h being sufficient to drive home safely following propofol sedation $[29,30]$. However, because this issue is one of the important medico-legal issues regarding sedation, we conservatively recommended that the amount of time a patient should wait before driving following propofol sedation is $\geq 24 \mathrm{~h}$.

\section{Sedation-related documentation}

Recommendation 28: Documenting of the monitored variables should be performed throughout all of the phases of propofol sedation at regular intervals (at least once every 5 min during sedation, and at least once every 15 min during recovery) (Evidence level: $D$, Recommendation grade: Class IIa). 


\section{Training in propofol sedation}

Recommendation 29: Both medical doctors in charge of sedation and monitoring medical personnel should have attended an accredited training course for propofol sedation (one that is accredited by an independent internal sedation committee or an external expert organization), and such a course should include both theoretical and practical aspects. Self-training in propofol sedation is strongly discouraged (Evidence level: B, Recommendation grade: Class I).

Recommendation 30: Qualification for basic life support (BLS) should be maintained via periodic participation in an accredited program for BLS, both by medical doctors and medical personnel in charge of sedation and monitoring (Evidence level: D, Recommendation grade: Class IIa).

Recommendation 31: A qualification (and maintenance thereof) in advanced cardiac life support (ACLS) is required for medical doctors in charge of sedation who will practice in locations where an ACLS provider is not immediately available (Evidence level: D, Recommendation grade: Class IIa).

Recommendation 32: The first clinical cases of propofol sedation performed by an individual should be supervised by an anesthesiologist or another doctor with previous experience of $>$ 100 propofol sedation cases (Evidence level: D, Recommendation grade: Class III).

\section{Commentary}

The doctor in charge of propofol sedation has the overall responsibility for sedation, and must have completed a theoretical and practical training course in propofol sedation. This includes pharmacological knowledge of propofol; familiarity with the various levels of sedation; and knowledge, recognition, and treatment of anticipated adverse events including cardiopulmonary resuscitation, maintaining open airways, intubation, and assisted ventilation $[3,5,27,31,32]$. Those monitoring medical personnel who will assist in propofol administration or perform patient monitoring must receive structured, relevant training in propofol sedation and basic airway management.

In this regard, the KMA recommends fulfilling a prior educational program for all of the practitioners responsible for propofol sedation. The KMA will prepare an official educational program (a $3 \mathrm{~h}$ theoretical training course and a $1 \mathrm{~h}$ ethical and legal lecture) in collaboration with the KSA. We believe that such a KMA policy will enhance patient safety in propofol sedation, although it lacks a practical training course and educational programs for other medical personnel.

\section{Issues relating to the abuse potential of propofol}

Recommendation 33: Propofol sedation should not be per- formed for therapeutic purposes. In some patients, repeated propofol sedation over a relatively short period of time may lead to psychological dependence. Therefore, in cases of repeated propofol sedation over a relatively short period, the practitioner should carefully evaluate the presence of psychological dependence on propofol and inform the patient that it is a potential risk (Evidence level: C, Recommendation grade: Class IIa).

\section{Commentary}

The first reported case of propofol abuse was in 1992 [33], and many abuse cases of propofol among healthcare providers have been reported since that time. In contrast to other countries, a significant portion of propofol abuse in Korea has been documented among laypeople [34]. Thus, it was eventually designated as a controlled substance in Korea in February 2011.

The abuse potential of propofol is strongly supported by molecular, animal, and clinical pharmacological data [34]. It appears to result from psychological dependence, rather than physiological dependence. Thus, when a patient receives propofol sedation repeatedly over a relatively short period of time, it is important for the practitioner to inform the patient of the potential for propofol dependence, and to carefully assess the presence of a psychological dependence on propofol. To date, only $\mathrm{Xu}$ et al. [36] has evaluated the efficacy of propofol sedation for its therapeutic effects (in their study, refractory chronic primary insomnia). However, considering the cardiopulmonary risks of short-term use of propofol and the potential for dependence in longer-term use, propofol sedation itself should not be used for therapeutic purposes.

\section{Acknowledgments}

The development of the guidelines was supported by a grant from the Clinical Research Infrastructure Composition Project (project title: Patient Safety Policy Research, H115C0988) from the Korean Ministry of Health and Welfare.

\section{ORCID}

Hyun Kang, http://orcid.org/0000-0003-2844-5880

Duk Kyung Kim, http://orcid.org/0000-0002-6555-2100

Yong-Seon Choi, http://orcid.org/0000-0002-5348-864X

Young-Chul Yoo, http://orcid.org/0000-0002-6334-7541

Hyun Sik Chung, http://orcid.org/0000-0001-7527-1866 


\section{References}

1. Hong SJ, Kang YJ, Jeon YH, Son JS, Song JH, Yoo CS, et al. Analysis of expert consultation referrals to the Korean Society of Anesthesiologists (KSA): a comparison of procedural sedation and general anesthesia. J Anesth 2013; 27: 218-23.

2. Roh WS, Kim DK, Jeon YH, Kim SH, Lee SC, Ko YK, et al. Analysis of anesthesia-related medical disputes in the 2009-2014 period using the Korean Society of Anesthesiologists database. J Korean Med Sci 2015; 30: 207-13.

3. American Society of Anesthesiologists Task Force on Sedation and Analgesia by Non-Anesthesiologists. Practice guidelines for sedation and analgesia by non-anesthesiologists. Anesthesiology 2002; 96: 1004-17.

4. Vargo JJ, Cohen LB, Rex DK, Kwo PY. Position statement: nonanesthesiologist administration of propofol for GI endoscopy. Am J Gastroenterol 2009; 104: 2886-92.

5. Dumonceau JM, Riphaus A, Schreiber F, Vilmann P, Beilenhoff U, Aparicio JR, et al. Non-anesthesiologist administration of propofol for gastrointestinal endoscopy: European Society of Gastrointestinal Endoscopy, European Society of Gastroenterology and Endoscopy Nurses and Associates Guideline--Updated June 2015. Endoscopy 2015; 47: 1175-89.

6. Eddy DM. Clinical decision making: from theory to practice. Designing a practice policy. Standards, guidelines, and options. JAMA 1990; 263: 3077, 3081, 3084.

7. Singh H, Poluha W, Cheung M, Choptain N, Baron KI, Taback SP. Propofol for sedation during colonoscopy. Cochrane Database Syst Rev 2008; (4): CD006268.

8. McQuaid KR, Laine L. A systematic review and meta-analysis of randomized, controlled trials of moderate sedation for routine endoscopic procedures. Gastrointest Endosc 2008; 67: 910-23.

9. Wang D, Chen C, Chen J, Xu Y, Wang L, Zhu Z, et al. The use of propofol as a sedative agent in gastrointestinal endoscopy: a meta-analysis. PLoS One 2013; 8: e53311.

10. Bo LL, Bai Y, Bian JJ, Wen PS, Li JB, Deng XM. Propofol vs traditional sedative agents for endoscopic retrograde cholangiopancreatography: a meta-analysis. World J Gastroenterol 2011; 17: 3538-43.

11. Sethi S, Wadhwa V, Thaker A, Chuttani R, Pleskow DK, Barnett SR, et al. Propofol versus traditional sedative agents for advanced endoscopic procedures: a meta-analysis. Dig Endosc 2014; 26: 515-24.

12. Conway A. Is sedation by non-anaesthetists really safe? Br J Anaesth 2014; 112: 583-4.

13. Perel A. Non-anaesthesiologists should not be allowed to administer propofol for procedural sedation: a Consensus Statement of 21 European National Societies of Anaesthesia. Eur J Anaesthesiol 2011; 28: 580-4.

14. Shergill AK, Ben-Menachem T, Chandrasekhara V, Chathadi K, Decker GA, Evans JA, et al. Guidelines for endoscopy in pregnant and lactating women. Gastrointest Endosc 2012; 76: 18-24.

15. Hepner DL, Castells MC. Anaphylaxis during the perioperative period. Anesth Analg 2003; 97: 1381-95.

16. Harper NJ. Propofol and food allergy. Br J Anaesth 2016; 116: 11-3.

17. Godwin SA, Burton JH, Gerardo CJ, Hatten BW, Mace SE, Silvers SM, et al. Clinical policy: procedural sedation and analgesia in the emergency department. Ann Emerg Med 2014; 63: 247-58.e18.

18. Lee CK, Dong SH, Kim ES, Moon SH, Park HJ, Yang DH, et al. Room for quality improvement in endoscopist-directed sedation: results from the first nationwide survey in Korea. Gut Liver 2016; 10: 83-94.

19. Enestvedt BK, Eisen GM, Holub J, Lieberman DA. Is the American Society of Anesthesiologists classification useful in risk stratification for endoscopic procedures? Gastrointest Endosc 2013; 77: 464-71.

20. Frieling T, Heise J, Kreysel C, Kuhlen R, Schepke M. Sedation-associated complications in endoscopy--prospective multicentre survey of 191142 patients. Z Gastroenterol 2013; 51: 568-72.

21. Cohen LB, Ladas SD, Vargo JJ, Paspatis GA, Bjorkman DJ, Van der Linden P, et al. Sedation in digestive endoscopy: the Athens international position statements. Aliment Pharmacol Ther 2010; 32: 425-42.

22. Igea F, Casellas JA, González-Huix F, Gómez-Oliva C, Baudet JS, Cacho G, et al. Sedation for gastrointestinal endoscopy. Endoscopy 2014; 46: 720-31.

23. Qadeer MA, Vargo JJ, Dumot JA, Lopez R, Trolli PA, Stevens T, et al. Capnographic monitoring of respiratory activity improves safety of sedation for endoscopic cholangiopancreatography and ultrasonography. Gastroenterology 2009; 136: 1568-76.

24. van Loon K, van Rheineck Leyssius AT, van Zaane B, Denteneer M, Kalkman CJ. Capnography during deep sedation with propofol by nonanesthesiologists: a randomized controlled trial. Anesth Analg 2014; 119: 49-55.

25. Weaver J. The latest ASA mandate: CO(2) monitoring for moderate and deep sedation. Anesth Prog 2011; 58: 111-2.

26. Standards of Practice Committee of the American Society for Gastrointestinal Endoscopy, Lichtenstein DR, Jagannath S, Baron TH, Anderson MA, Banerjee S, et al. Sedation and anesthesia in GI endoscopy. Gastrointest Endosc 2008; 68: 815-26.

27. Riphaus A, Wehrmann T, Weber B, Arnold J, Beilenhoff U, Bitter H, et al. S3-guidelines--sedation in gastrointestinal endoscopy. Z Gastroenterol 2008; 46: 1298-330.

28. Leslie K, Clavisi O, Hargrove J. Target-controlled infusion versus manually-controlled infusion of propofol for general anaesthesia or sedation in adults. Cochrane Database Syst Rev 2008; (3): CD006059.

29. Horiuchi A, Nakayama Y, Fujii H, Katsuyama Y, Ohmori S, Tanaka N. Psychomotor recovery and blood propofol level in colonoscopy when 
using propofol sedation. Gastrointest Endosc 2012; 75: 506-12.

30. Horiuchi A, Nakayama Y, Kajiyama M, Kato N, Kamijima T, Ichise Y, et al. Safety and effectiveness of propofol sedation during and after outpatient colonoscopy. World J Gastroenterol 2012; 18: 3420-5.

31. Secretariat for Reference Programmes Gastroenterology Committee. Danish guideline for the Propofol sedation for gastroenterological, endoscopic procedures performed by non-anaesthetically-trained personnel - and associated training, applicable for the Capital Region of Denmark's general hospitals. Available from http://www.esgena.org/assets/downloads/pdfs/guidelines/2011_danish_propofol_guideline. pdf.

32. Training Committee. American Society for Gastrointestinal Endoscopy. Training guideline for use of propofol in gastrointestinal endoscopy. Gastrointest Endosc 2004; 60: 167-72.

33. Follette JW, Farley WJ. Anesthesiologist addicted to propofol. Anesthesiology 1992; 77: 817-8.

34. Kim EJ, Kim SH, Hyun YJ, Noh YK, Jung HS, Han SY, et al. Clinical and psychological characteristics of propofol abusers in Korea: a survey of propofol abuse in 38, non-healthcare professionals. Korean J Anesthesiol 2015; 68: 586-93.

35. Wilson C, Canning P, Caravati EM. The abuse potential of propofol. Clin Toxicol (Phila) 2010; 48: 165-70.

36. Xu Z, Jiang X, Li W, Gao D, Li X, Liu J. Propofol-induced sleep: efficacy and safety in patients with refractory chronic primary insomnia. Cell Biochem Biophys 2011; 60: 161-6. 